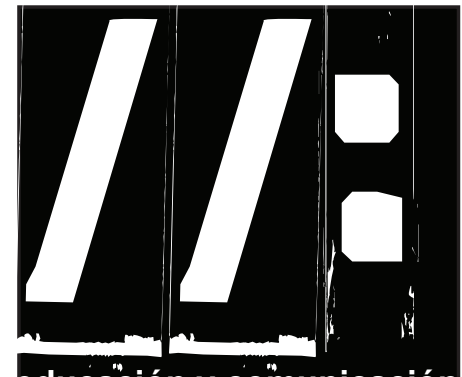

aduca"ción y cömunicación 10: 47-59 Mayo 2015

\title{
CAPACIDADES DELIBERATIVAS Y TIC, GLAVES EDUCATIVAS PARA El DESARROLLO DE LOS MENORES EN LAS PLENITUD DE SUS DERECHOS. EL PROYECTO GADE
}

\section{Deliherative capacities and ICT, education keys to the development of children in the fullness of their Rights. The GADE Project.}

\author{
Michel Santiago del Pino \\ Grupo de Investigación: Investigacion Educativa \\ "Eduardo Benot" (HUM 230) \\ Facultad de Ciencias de la Educación. Universidad de \\ Cádiz (España) \\ E.mail: michelsantiago@telefonica.net
}

Resumen:

Este artículo quiere dar a conocer el profundo alcance y la alta calidad de los resultados que un programa educativo puede obtener, cuando parte de la adecuada comprensión del nuevo paradigma de la niñez contenido en la Convención de los Derechos del Niño. El Proyecto Desarrollo de la Capacidad Deliberativa en Escolares (CADE), llevado a cabo en Costa Rica, aprovecha intensamente las tecnologías en el aula como instrumentos valiosos para el desarrollo de capacidades de pensamiento de orden elevado, para la exploración de vías comunicativas y para la producción de contenidos con capacidad de intervención y participación social. Evaluado como útil en todo tipo de contextos, el Proyecto CADE se ha revelado como una herramienta extremadamente eficiente en enclaves desfavorecidos, propiciando incluso beneficios a la comunidad. Finalmente, intentamos dar cuenta de la potencia global del Proyecto, a través de la experiencia que pudimos compartir en la Escuela Cacique Guarco.

Palabras clave: Educación y alfabetización mediática e informacional, capacidades deliberativas, Derechos del Niño, TIC, Proyecto CADE.

\section{Abstract:}

This paper aims to discover the profound extent and quality of the outcomes that an educational program can obtain, when it comes from the proper understanding of the new paradigm of childhood contained in the Convention on the Rights of the Child. The Deliberative Capabilities in School Age Children Project (CADE, its Spanish acronym), held in Costa Rica, heavily leverages technology in the classroom as valuable tools for developing thinking skills of a high order, to explore communication ways, and for content production capacity of intervention and social participation.Evaluated as useful in all kinds of contexts, CADE project has proved to be an extremely efficient tool in disadvantaged enclaves, including promoting community benefits. Finally, we try to account for the overall power of the Project through the experience that we shared on the Cacique Guarco School, on Cartago province. Keywords: Education and media and information literacy, deliberative capacities, Rights of the Child, ITC, CADE Project.

Recibido: 29/11/2014 Revisado: 19/1/2015 Aceptado: 27/2/2015 Publicado: 1/5/2015

https://dx.doi.org/10.25267/Hachetetepe.2015.v1.i10.5 


\section{II: Educación para la diversidad y comunicación}

\author{
"Avivar el espíritu crítico, desenmascarar la falsedad, \\ comprometerse con los más desfavorecidos, \\ combinar políticas eficaces de distribución \\ $\mathrm{y}$ de reconocimiento, \\ potenciar la verdadera educación que ayuda a pensar \\ y a convivir, movilizarse para salvar la infancia de la \\ violencia.. \\ Ese es el camino..." \\ Miguel Ángel Santos Guerra
}

\section{Introducción}

$\mathrm{D}$ esde 1924, los vaivenes históricos del siglo XX acompañaron el trabajo de consolidación de los Derechos del Niño, hasta que se acordara la Convención de las Naciones Unidas en el año 1989. Desde entonces, los Derechos del Niño han sido un marco que invocar, un referente al que acudir, un soporte en el que apoyar la defensa cuando niños o niñas sufren daño o un gran menoscabo en los Derechos informados. Hoy, con la ratificación de todos los países, a excepción de Estados Unidos y Somalia, se iza un instrumento con el estatus jurídico más alto a nivel internacional habido hasta la fecha en la materia, que debería ser utilizado con mayor asiduidad en todas sus dimensiones y dotando al Comité, que vela y evalúa su aplicación, con poderes coercitivos sobre los Estados firmantes, de los que sí gozan otros acuerdos internacionales.

Pero, dejando aparte el largo camino por recorrer, pocos han ido más allá de la utilidad, si bien grande, que presta su interpretación jurídica en el ámbito de la asistencia y de la protección. El acuerdo ratificado no solo es importante por su expansión en número de Derechos y por su influencia en la elaboración de las
Constituciones de muchos países firmantes, sino que también por la relevancia del cambio en la construcción social del discurso acerca de la esencia legítima del menor, como entidad colectiva y como ser humano con toda su potencia individual.

Rosario Carmona Luque, recientemente galardonada con el Premio de Ensayo en Derechos Humanos, otorgado por la Academia de Derechos Humanos y Derecho Internacional Humanitario de la American University, define la Convención de los Derechos del Niño (CDN en adelante) como un Tratado de Derechos Humanos en sentido estricto, porque no solo declara y categoriza sino que también "cohesiona el tratamiento de una materia hasta entonces dispersa, y lo hace imprimiéndole un alcance amplio y una perspectiva actual, respecto de la posición del niño como titular de derechos propios y al contenido de estos últimos" (Carmona Luque, 2011: 53).

Partiendo de la necesidad de interpenetración de todos los Derechos, y con la importancia que señala Carmona Luque, la Convención de 1989 ofrece también este ámbito de desarrollo que es de suma importancia, ya que su introducción supuso una inflexión crucial en la concepción de los y las menores como entidades autónomas, a través de la garantía de sus Derechos civiles y políticos: nombre y nacionalidad, libertad de expresión, de pensamiento, conciencia y religión, asociación, opinión del niño, protección de la vida privada, de participación de acuerdo con su capacidad y desarrollo (artículos, 7, 8, 12, 13, 14, 15, 16 y 17). Es decir, y contrastando con los descriptores utilizados en documentos anteriores (Dávila Balsera y Naya Garmendía, 2006: 86), no solo los y las menores dejan de ser objetos de protección, para ser Sujetos de Derechos, sino que consolida un discurso dignificante de la 
infancia desde la equidad de la responsabilidad social. La profesora Carmona Luque, enfocó su investigación de tesis previa al trabajo premiado, sobre el valor de la Convención como instrumento de progresividad (op.cit. 2011). Y en esta progresividad, trabaja activamente el Comité de los Derechos del Niño aportando ampliaciones al texto original. En esa dirección, la Asamblea General de las Naciones Unidas aprobó, en diciembre de 2011, el Protocolo Facultativo de la Convención sobre los Derechos del Niño relativo a un procedimiento de comunicaciones, que recientemente, enero de 2014, ratificó el Estado español.

Por medio de ese Protocolo, si un niño cree que sus derechos fundamentales fueron violados, podrá presentar una queja al Comité, por sí mismo, a través de sus tutores, o de una de las ONG validadas para ello. Éste es un avance de gran importancia, porque desde el reconocimiento del menor como sujeto competente en los asuntos de su propio interés, hasta la entrada en vigor del Protocolo, no existía la concreción del Derecho a través de un cauce de expresión legal para los menores a título individual. Con este instrumento, la Convención de los Derechos del Niño reafirma "la condición del niño como sujeto de derechos y ser humano con dignidad y con capacidades en evolución”, y da carta de realidad a uno de sus Principios Generales "la atención a la opinión del niño en todos los asuntos que lo afecten".

Esta falta de consideración real de la opinión de los y las menores se aprecia en todos los contextos que les afectan y, de forma especial, en aquellos en los que están presentes como colectivo, esto es principalmente el sistema educativo.

Santos Guerra recuerda haber oído a Francesco Tonucci que "la escuela es una institución ilegal porque quebranta sistemáticamente la ley que exige que los niños y niñas sean consultados sobre aquellas cuestiones que les conciernen" (Santos Guerra, 2014: 216). Es cierto, las instituciones a cuyas exigencias las y los menores dedican entre ocho y diez horas de su vida diaria, de media, no les tienen en cuenta en lo que a ellos respecta. Sin embargo, coincidimos plenamente con el lúcido Catedrático de Didáctica en que "la escuela puede ser un camino hacia la libertad a través del conocimiento y de la convivencia" (op.cit.2014: 216). Por ello el objetivo prioritario de este artículo es dar evidencia de cuan posible es integrar los Derechos del Niño en la práctica educativa, con la presentación de un proyecto con cimientos sólidos y vanguardista, que se nutre de la diversidad con un fuerte sentido de la justicia, para centrarse en extraer la voz individual y la acción colectiva de los menores desde procesos rigurosos de deliberación, hasta alcanzar una calidad reflexiva y cooperativa rara de ver en el mundo adulto, ofreciendo así la oportunidad a los niños y niñas de madurar con el sentimiento feliz y consciente de poseer unas capacidades singulares y valiosas, con las que aportar recursos y perspectivas útiles en la toma de decisiones para la mejora de la sociedad democrática.

\section{El Proyecto Desarrollo de la Capacidad Deliberativa en Escolares (CADE)}

A pesar de la tardanza en cristalizar legalmente el espacio para la voz de los y las menores, las palabras del texto de la CDN han sido activas desde el mismo momento de su publicación universal, como nuevo paradigma de la niñez. Mary Carlson y Felton Earls (Profesores de la Escuela de Salud Pública de la Uni- 


\section{II: Educación para la diversidad y comunicación}

versidad de Harvard), apreciaron su verdadero significado "El nuevo estado revolucionario de los niños como "titulares de derechos" los dota del respeto, la igualdad, las garantías jurídicas de protección por parte de actores estatales y no estatales, así como su reconocimiento como agentes sociales". Y coincidieron con el sociólogo Karl-Eric Knutsson en la necesidad de "ir más allá de los enfoques de caridad de las 'causas nobles' hacia la visión post-CDN de 'ciudadanos dignos"” (Carlson y Earls 2011: 224).

Esta visión, desviando el foco desde las carencias de los menores hacia sus capacidades emergentes, cimentó su experiencia de trabajo a través de proyectos cuyo fin era crear las condiciones para el ejercicio de los derechos de participación de los adolescentes. El interés de ambos doctores se acrecentó en torno a la 'democracia deliberativa', como contexto idóneo para el desarrollo de competencias de los niños como ciudadanos. La teoría y la investigación fundamentadas en Amartya Sen y Jürgen Habermas, entre otros, generaron un modelo vertebrado, de una parte, por el reconocimiento de la agencia y las capacidades del niño, concebidas como estados positivos y optimistas de ser, sin ignorar las causas y consecuencias de privaciones de capacidad y, por otra, por la comunicación como fuente dinámica y continuada de socialización desde la integración social, a través de sus Derechos de opinión y participación dentro de la comunidad.

Sobre dicho modelo, pusieron en marcha su Programa de Jóvenes Ciudadanos (Young Citizens Program,) que comenzó con grupos deliberativos a pequeña escala en Chicago, madurado hasta convertirse en un grupo ensayo controlado aleatorio en el norte de Tanzania, evaluado rigurosamente. El éxito de estos programas, junto a sus estudios sobre violencia y bienestar social en la niñez y la adolescencia, les llevó a considerar que se trataba de un enfoque intelectualmente más estimulante para los menores que aquellos otros que enfatizan los riesgos y los factores de protección en la infancia, y alentados por ello buscaron un territorio propicio para la implementación y desarrollo del modelo, con vocación de permanencia. Costa Rica ofrecía un contexto muy adecuado, no en vano sus 4.7 millones de habitantes gozan de una idiosincrasia penetrante y particular, que tiene entre sus preocupaciones sociales principales la mejora del sistema público de educación. Con una tasa de alfabetización cercana al $95 \%$, hace ya décadas que el país viene haciendo un gran esfuerzo hasta poder dedicar a la educación el $7.20 \%$ del producto interior bruto (PIB), un 11,3\% más que lo invertido en el curso lectivo de 2013 (tengamos en cuenta la continua bajada en España hasta el 4,37\% del PIB, y que el Ejecutivo de Rajoy se comprometió con Bruselas a rebajar hasta el 3,9\% para 2015). Más lejos, Costa Rica debe alcanzar el $8 \%$ del PIB en inversión para la educación en los próximos años, de acuerdo a una reforma constitucional aprobada en 2011.

Por ello, no fue casual que coincidiendo en el tiempo, 2001, el Departamento de Investigación de la Fundación Omar Dengo (FOD), y en línea con su anticipación preclara del potencial educativo de las tecnologías, estuviera por entonces estudiando el modo de imbricar su apropiación con el desarrollo cognitivo y social de la infancia. Sobre la perspectiva teórica de Carlton y Earls, CADE acuñó el concepto 'deliberación' "como un diálogo cooperativo dirigido a la resolución de problemas y conflictos de carácter público, basado en la detenida consideración de la información disponible, en la toma de perspectiva, en la participa- 
ción equitativa, en la construcción de acuerdos a partir de las aportaciones de todos y en la búsqueda del bien común.” (Fundamentos Teóricos. Proyecto CADE, Fundación Omar Dengo). Además de las bases teóricas, los doctores de Harvard aportaron la elaboración de un diagrama de los componentes de desarrollo de las capacidades deliberativas (Earls y Carlson 2002), en el que, a partir de la traducción práctica y la aplicación de la acción y la ética del discurso comunicativo, combinaron el modelo de capacidades con competencias comunicativas, estableciendo cuatro principios a los que tender en la práctica deliberativa:

Confianza: entendida como la conciencia de las posibilidades y situaciones del otro, y de la oportunidad de compartir un marco ético para la acción social.

Toma de perspectiva: entendida como la comprensión de los sentimientos y emociones del otro, y las razones de su conducta.

Entendimiento mutuo: entendido como la toma de acuerdos alcanzados mediante el diálogo, a través del pensamiento crítico y la aplicación de procesos de pensamiento científico que permitan por un lado determinar la autenticidad, precisión y valor de una información; $y$, por otro tomar perspectiva, para generar tolerancia, poder superar las posiciones encontradas y lograr un saber común fundado en un acuerdo reconocido como válido y, por tanto, vinculante.

Acción social compartida: entendida como acción prosocial orientada por normas y valores que el grupo reconoce como válidos. Implica cooperación, coordinación y regulación.

En este marco de definición, se formularon cuatro grandes competencias para la deliberación, que permiten operativizar las capacidades para la deliberación, a efectos de su aprendizaje y evaluación. (Bujanda, 2005: 7)
- Iniciar una deliberación, reconociéndose como agente social y como miembro de una comunidad local, nacional, regional y global; con derecho a participar en la vida política y cívica, y con conciencia de las responsabilidades que puede asumir; con capacidad de iniciativa y autonomía para resolver los problemas que les aquejan o de mejorar sus circunstancias, sin estar supeditados a las personas adultas

- Desarrollar diálogos de alta calidad, discriminar qué es un buen diálogo y ser conscientes de su sentido e importancia; poseer ciertas habilidades dialógicas, como la capacidad de escuchar de manera activa y empática, y de expresarse con claridad, sinceridad, pertinencia, coherencia y concisión; considerar en los argumentos el punto de vista de los demás para determinar lo que es justo; buscar la participación equitativa y las posiciones simétricas para el diálogo, desde el respeto mutuo y la comprensión de la igualdad y la dignidad de los seres humanos.

- Idear propuestas de solución a problemas de la comunidad, lo cual supone problematizar de un modo crítico las necesidades y problemas de la comunidad (local o nacional), y asumirlos como una responsabilidad individual y colectiva; informarse sobre la realidad y sopesar detenidamente la información y los distintos puntos de vista involucrados; aplicar la imaginación y la creatividad a la hora de explorar posibles soluciones a los problemas; analizar diferentes alternativas y sus consecuencias desde la toma de perspectiva y el respeto a las diferencias; hacer uso de los cauces para participar y generar un impacto en la vida comunitaria.

- Construir acuerdos de cooperación, elaborar 


\section{II: Educación para la diversidad y comunicación}

criterios racionales con miras a lograr un acuerdo para la resolución de conflictos; participar en la toma colectiva de decisiones aceptables para todos, con base en procedimientos orientados al acuerdo; actuar con asertividad desde la responsabilidad y el cuidado de uno mismo y de los demás; asumir la responsabilidad por las decisiones tomadas y saber respetar los acuerdos.

La FOD, en la persona de $\mathrm{M}^{\mathrm{a}}$ Eugenia Bujanda, acometió el desarrollo de 'medidas de autoeficacia para la deliberación', así como las estrategias de uso de las tecnologías digitales y de los recursos del sitio web y, junto a los docentes del Programa Nacional de Informática Educativa MEP-FOD (en adelante PRONIE), pusieron a punto el diseño curricular y una primera aplicación piloto de la propuesta diseñada, que incluía una evaluación formativa que sugiriera posibles mejoras, y una primera aproximación a los resultados. Finalmente se incorporó al diseño una guía didáctica dirigida a orientar a los educadores en todos los aspectos de la puesta en práctica. Esta guía está compuesta por cinco unidades de aprendizaje, más una sexta dedicada a realizar una evaluación final.

CADE emergió como un proyecto de grandes expectativas sobre las capacidades de los niños para ejercer su derecho a acceder y a emitir información, a expresar sus pensamientos y sentimientos, a ser escuchados y a participar en las decisiones que les afectan. Y, además, fue muy ambicioso, tanto metodológicamente como con respecto al papel desempeñado por las tecnologías, cuya explotación se contempla en una doble dimensión: como instrumento para la creación de contenidos audiovisuales y de aplicaciones a través de la computación y como instrumento de educación mediática e informacional, aprovechando su ilimita- do potencial para vehicular procesos comunicativos y analíticos. Es lógico que dicho nivel de exigencia se acompañara en sus inicios con cierto escepticismo. Sin embargo, "cuando se constató el asombroso impacto que tienen en los niños los ambientes tecnológicamente ricos y significativos; y cuando, en una reunión con los jueces del Tribunal Superior de Elecciones (la instancia judicial responsable de monitorear los procesos electorales y las prácticas democráticas en Costa Rica), estos sugirieron que el proyecto no debía empezar en la escuela primaria, sino en la etapa preescolar" (Bujanda 2005:2, recogido en Carlson, Earls y Fonseca, 2002), el ánimo se redobló, efecto que no ha dejado de crecer a la par que evoluciona su aplicación.

Desde entonces se han beneficiado de CADE cerca de 2000 niños y niñas de 10 a 13 años que cursan $5^{\circ}$ y $/ 06^{\circ}$ grado en escuelas públicas, Con una perspectiva pedagógica construccionista y trabajando con el enfoque experiencia-acción a través del Aprendizaje por Proyectos, en la actualidad los y las estudiantes de ocho escuelas, que son como premisa de distintos niveles socioeconómicos y entornos culturales, desarrollan un proyecto de investigación sobre temas curriculares que incorporan simulaciones, representaciones y animaciones elaboradas por medio de la programación. El trabajo desarrollado es de tipo colaborativo, por lo cual se trabaja siempre en equipos llamados 'compañías de producción'.

Además de una formación inicial de 40 horas para activar su rol de guía y mediación, y capacitaciones anuales de actualización sobre las novedades en el diseño de la propuesta, los docentes disponen de guías y manuales, así como de materiales y recursos complementarios. También disfrutan de un acompañamiento 
permanente con visitas de los asesores de la FOD y el PRONIE. Por otro lado, disponen de una comunidad virtual donde transfieren sus hallazgos y comparten los materiales que ellos mismos han elaborado. A la transferencia virtual de resultados, suman varias reuniones al año donde los docentes y responsables analizan los avances e intercambian ideas.

\section{En la Escuela Cacique Guarco.}

Las limitaciones del presente documento impiden profundizar en la riqueza de los fundamentos, metodología e implementación que ha mantenido desde sus inicios el Proyecto CADE. Para una aproximación, se puede comenzar por las referencias bibliográficas aportadas, muchas disponibles en línea. Por su parte, la Fundación Omar Dengo brinda la posibilidad de conocerlo al detalle en su página web, así como guías y actividades sumamente útiles. Sin embargo, sí podemos resumir aquí la experiencia directa que, gracias a la invitación de la Fundación Omar Dengo, hemos tenido la oportunidad de compartir en la Escuela Cacique Guarco. Se trata de una Escuela Pública de Educación de Atención Prioritaria, ubicada en El Tejar de El Guarco entre los diques y el Parque industrial de la provincia de Cartago en Costa Rica. El motivo del encuentro fue presentar la evolución del Proyecto a Maya Carlson y Felton Earls, así como a las personas involucradas en él: $\mathrm{M}^{\mathrm{a}}$ Eugenia Bujanda (hoy directora del PRONIE), diseñadora del proyecto y responsable de su puesta en marcha, y Natalia Rodríguez, responsable actual de CADE. También nos acompañó María Esther Rojas como asesora pedagógica del Programa Nacional de Informática Educativa, y quien pertenece al grupo que actúa de nexo entre esta zona educativa, el Ministerio de Educación Pública y la FOD.

Dentro de la flexibilidad para ajustar las guías a las características de su grupo y de la autonomía que el Proyecto concede a las y los maestros que lo implementan, Xinia Zambrana, maestra encargada de CADE en la escuela, explica como, de forma previa a la investigación y selección de los temas más preocupantes para los estudiantes, la propia evolución del Proyecto puso de manifiesto la necesidad de acometer las dos primeras unidades didácticas: la Identidad Ciudadana y la Integridad humana. La primera de ellas parte de la pregunta ¿Yo también puedo ser ciudadano? Junto a la maestra, veinte niños de $5^{\circ}$ curso sentados en óvalo replican ante nosotros los resultados del proceso deliberativo seguido, hasta obtener un consenso de respuesta. Primero nos presentan a los menores encargados, en esa ocasión, de tres funciones: el Guardián de Turnos, el Fotógrafo y el Anotador. La atribución de roles se promueve en CADE para acentuar el sentido de la responsabilidad. Y comenzamos el visionado de un cortometraje, elemento a partir del que se proponen y guían los contenidos de debate. Hoy sirve para introducir las preguntas con las que refrescan las conclusiones obtenidas en el proceso deliberativo. Tras unos breves minutos en los que Xinia ánima a intervenir, la timidez ha sido vencida sin esfuerzo y, sin excepciones, los niños levantan las manos masivamente, todos quieren participar con un grado de excitación controlado, que les permite una atención intensa sobre el proceso que se está desarrollando. Los niños enlazan ideas una tras otra hasta alcanzar un número importante y variado de atribuciones que, en su opinión, dotan de identidad a una persona. Finalmente, ellos mismos se comprenden y afirman como ciudadanos. 
Durante el descanso los niños se agrupan en torno a los visitantes con una actitud completamente inesperada. De forma desinhibida, asertiva y afectiva entablan conversación y pronto consiguen dirigirla hacia sus centros de curiosidad: cómo nos llamamos, de dónde somos, las peculiaridades de nuestro acento, o lengua en el caso de los angloparlantes,... y qué sabemos del tico recién incorporado como portero del Real Madrid. El grado de resolución y control de la situación son propios de un perfil de maduración difícil de encontrar entre los estudiantes españoles, sobre todo de forma tan unánime. Después de mostrarnos algunas habilidades personales, la sesión prosigue de vuelta a sus asientos, esta vez frente a los ordenadores. Van a continuar con el diseño que están programando con Scractch. Xinia aventura que el avance de hoy se hará sobre "otro reto pequeñito": "Vamos a cambiar el escenario y los colores, y luego a programar la secuencia. Nos vamos a fijar en el orden". La actividad les absorbe la atención hasta el punto de que hemos perdido totalmente el interés para ellos. A la despedida, nos regalan de nuevo entre sonrisas su gran simpatía.

Compartimos la segunda parte de la visita con la otra clase de $5^{\circ}$. Las habilidades sociales de estos alumnos no desmerecen del grupo anterior. En esta ocasión nos ofrecen los resultados de la segunda unidad didáctica, construida por la maestra. El vídeo con el que fueron llamados a la reflexión exhibe una cadena de favores entre desconocidos en un barrio urbano. Los niños y niñas recuerdan rápidamente a lo que se refiere el concepto que estuvieron trabajando "Integridad Humana": "tiene que ver con la relación con los demás", "no hay que maltratar a las personas", "es bueno hacer amigos", "hay que decir no a la violencia", "y al bullying", "Tenemos que respetarnos los unos a los otros", "Y saber compartir, sin pelearnos" - ¿Y cómo?, pregunta Xinia?- "Respetando los valores de los demás", "siendo amables", "siendo honestos"...

Durante la segunda parte de la sesión, la clase quiso mostrarnos una experiencia vivida a través del Proyecto, de la que la Escuela se siente especialmente orgullosa. Tres años atrás, durante el proceso de deliberación en torno a los problemas de la comunidad, emergió con fuerza su situación medioambiental como la mayor de sus preocupaciones. El alumnado de este centro procede de las familias asentadas en 'tugurios' (chabolas) levantadas a orillas del río Guarco. Una vez que entre todos determinaron el foco principal en la suciedad de las aguas, debido a los vertidos desde las viviendas, los niños salieron a la captura de imágenes testimoniales. Con ellas, montaron una presentación muy explícita, de la que se sirvieron para dar a conocer su opinión a la comunidad. ¿Qué más podían hacer, desde su identidad como ciudadanos íntegros con capacidad de agencia e influencia en el entorno? Dar el último paso. Se dirigieron al Ayuntamiento y denunciaron el caso. Cerraron así el ciclo educativo como ciudadanos copartícipes en la construcción de una sociedad democrática. Dos años después, el barrio goza de una planta depuradora de aguas con la que se ha dado fin al problema. Aún sin tener la total certeza, es del todo factible que la voz del alumnado de primaria de Cacique Guarco hiciera valer su opinión, bien informada, influyendo en la toma de decisiones políticas que les afectan.

Las conductas concretas de la maestra se ajustaron a las definidas por CADE como adecuadas en los tutores. Se trata de un estilo de mediación caracterizado por la horizontalidad; la aceptación, la no-discri- 
minación y el apoyo; el fomento de la tolerancia, la ecuanimidad y del rechazo al prejuicio; el fomento de la participación equitativa; el fomento de la apertura al diálogo y la escucha; el fomento de la conciencia de ser sujeto de derechos cívicos y políticos; el fomento de la capacidad comunicativa; el fomento de la argumentación razonada; el fomento de la toma de perspectiva, el establecimiento de relaciones entre ideas y la integración de perspectivas; el fomento de la construcción de acuerdos aceptables para todos; y el manejo no directivo, justo y promotor de la autorregulación de los conflictos interpersonales (Bujanda, 2005: 18). Xinia Zambrana usa además el tejido capaz de unir todos estos elementos: un escrupuloso respeto cargado de afecto.

\section{¿Expectativas en España?}

En una dirección diferente de la planteada por la Unión Europea desde el desarrollo de competencias para futuras demandas laborales, y de responsabilidades de participación política y cívica en su etapa adulta, el Proyecto CADE se concibe como un proceso para facilitar el ejercicio de los Derechos de Expresión, de Opinión y de Participación que los niños y adolescentes ya tienen en el presente desde la promulgación de los Derechos del Niño en 1989 (Bujanda, 2007; Fundación Omar Dengo, 2010). Este es a nuestro modo de ver uno de los grandes aciertos basales del Proyecto CADE, porque transforma a los menores, de objetos receptores de contenidos, en sujetos activos dignos del mayor de los respetos, que se expresa a través de la valoración positiva de todos sus aportes al colectivo del grupo y a la comunidad en la que crecen y/o se desenvuelven.
No se trata pues de que el niño se imagine a sí mismo en un mundo futuro y las competencias que necesitará en él, idea o fantasía cuya concreción real resulta improbable y que exige una capacidad vidente ausente por el momento entre las humanas. No se arroja, por tanto, al menor a vincularse a esa proyección de sí mismo, en la que le es dicho que encontrará una justificación a sus esfuerzos de formación. Por el contrario, se pone el foco sobre su desarrollo potencial aprovechando la intensa capacidad de 'mindfullnes' propia de la infancia. Se pretende captar toda su atención al aquí y al ahora, en territorio franco. Se trata de que se cumpla su derecho a ser educado como ser pensante con asombrosas capacidades críticas y de resolución de problemas, y lo pueda hacer gracias a la deliberación junto a sus pares y a su comunidad, a través de las herramientas, básicas en nuestro tiempo, de la educación mediática e informacional.

En la actualidad, la intensa interacción de los menores con las nuevas tecnologías los sitúa en el mismo rango que a los adultos como productores-consumidores, 'prosumers' en términos de Alvin Tofler. Sin embargo, y a nuestro modo de ver, la potencia de los y las menores como emisores de actos comunicativos con capacidad para intervenir en los procesos que les afectan, que el Protocolo reseñado más arriba consolida, debería modificar la actitud pedagógica de los docentes para transformarse en guías conductores del desarrollo de sus capacidades críticas y deliberativas. La falta de agilidad y/o voluntad política y educativa para responder a las necesidades, eventuales o perseverantes, de los y las menores como sujetos de Derechos repercute profundamente en la madurez de la sociedad, no solo para apercibirse de cuando estos son transgredidos, y se ha de actuar de modo sensible y 


\section{II: Educación para la diversidad y comunicación}

diligente, sino para lograr menores educados en plenitud y dotados de las herramientas más importantes para construir una sociedad auténticamente democrática en su etapa adulta.

En España, a pesar de las buenas intenciones y del convencimiento de la incidencia de las tecnologías en la motivación de los menores, aún se desconfía de los modelos de integración

«Hoy podríamos definir las pedagogías emergentes como el conjunto de enfoques e ideas pedagógicas, todavía no bien sistematizadas, que surgen alrededor del uso de las TIC en educación y que intentan aprovechar todo su potencial comunicativo, informacional, colaborativo, interactivo, creativo e innovador en el marco de una nueva cultura del aprendizaje» (Adell y Castañeda, 2012: 15).

Esas experiencias "no bien sistematizadas", dan cuenta de que, en nuestro país, las iniciativas se ponen en marcha a instancias del compromiso personal de docentes concienciados que, además, no gozan de ningún apoyo institucional. Y más lejos, en 2012 se llevó a cabo el estudio más amplio hasta la fecha (fue cumplimentado por un total de 5.161 sujetos), acerca de la visión y prácticas del profesorado de $5^{\circ}-6^{\circ}$ de Educación Primaria y $1^{\circ}-2^{\circ}$ de E.S.O, en torno al programa 2.0, y de aquellos docentes de los programas TIC específicos de Madrid y Valencia. Entre sus conclusiones, señala que "los materiales didácticos tradicionales (como son los libros de texto y las pizarras) siguen siendo los recursos más empleados en las aulas Escuela 2.0. a pesar de la abundancia de la tecnología digital. La mayor parte del profesorado (en porcentajes entre el 80-60\%) indica que los tipos de actividades que desarrolla en el aula con TIC de forma más repetida son: la demanda al alumnado de búsqueda de información, la realización de trabajos con procesadores de textos, la cumplimentación por los estudiantes de ejercicios on line, y el ofrecer explicaciones a través de la PDI (Pizarra Digital Interactiva). Por el contrario, la inmensa mayoría (más del 70\%) reconoce que no elabora materiales digitales on line, que no solicita a los estudiantes tareas de publicación en la red, y que no desarrolla proyectos telemáticos entre clases. (Area, 2012: 213).

Una vez que en 2012 se suprimiera el Programa 2.0, la reducción económica próxima al $60 \%$ ha limitado drásticamente los objetivos centrados en la integración de las nuevas tecnologías en los procesos de enseñanza-aprendizaje. El foco de los programas TIC actuales está en el desarrollo de infraestructuras para interoperabilidad entre los Sistemas de Gestión (ERP) de las distintas administraciones educativas. En consecuencia, la supervivencia de los Entornos Virtuales de Aprendizaje (EVA), se cifra en el desarrollo de, lo que denominan desde el Ministerio de Educación, modelos próximos al BYOD (Bring Your Own Device), 'Trae Tu Propio Aparato'. Suponemos que se refiere a los aparatos que los padres hayan podido adquirir para sus hijos y que, buenamente, les van a permitir llevar a la escuela. Esto, en buena lógica, establece una desigualdad de partida. Podrán aparecer en el aula móviles, tabletas, ordenadores,... soportes en fin de variada tipología, con lo que aparece la necesidad de contar con una referencia común para dispositivos de alumnos y profesores, que garantice su correcto funcionamiento en el ámbito escolar. Por su parte, la página de Recursos, indica que existen en línea multitud de ellos y muchos gratuitos, y anima a los docentes a producir materiales y compartirlos virtualmente. (Fuente: Página web del Instituto Nacional de Tecnologías Educativas y de Formación del Profesorado INTEF, Ministerio de Educación). 


\section{Conclusiones}

A pesar de las expectativas sumamente negativas en España, consideramos que el Proyecto CADE ofrece grandes posibilidades de implementación en nuestro país. De hecho, su aplicación en Costa Rica se hace con el uso del ordenador compartido. Un gran aporte ofrece también el currículum de Alfabetización Mediática e Informacional (AMI) promovido por la UNESCO, donde se contempla la posibilidad de llevar a cabo la integración de la Educación Mediática, y el consiguiente desarrollo del pensamiento crítico, a partir de cualquier soporte de contenidos comunicativos extraídos de la realidad actual. Así, la prensa, la televisión, los vídeos y videojuegos, etcétera. Tal vez algo como CADE aún no se pueda desarrollar al completo, pero ampliar los objetivos AMI al desarrollo de las capacidades deliberativas de los estudiantes de cualquier nivel, con la ayuda del fundamento, metodología, de las guías y manuales de CADE, puede enriquecer el aprendizaje de todas las materias y está al alcance de cualquier docente interesado. Por otra parte, las tesis de Carlson y Earls forman parte de un potente movimiento internacional que impulsa el reconocimiento y la defensa de los derechos de participación, que constituyen el aspecto más retador del nuevo paradigma de la niñez proveniente de la Convención. A este movimiento se han unido organismos internacionales como UNICEF y el Consejo de Europa.

«Es la primera de estas tres [las 'tres P' de la Convención: participación, provisión y protección] la que ha constituido el aspecto más desafiante del articulado de la Convención, y la que ha sido abordada por muchos investigadores y practicantes de todo el mundo que valoran la libertad de los niños ciudadanos de hoy y que se han dado cuenta de que las responsabilidades del mañana serán de ellos» (Holden y Clough, 1998: 9)

Lo queramos o no, las herramientas tecnológicas nos pertenecen y están ya inscritas en nuestro acervo histórico y social contemporáneo. Sostener el paradigma educativo que alienta la reproducción memorística de contenidos y la búsqueda de resultados de respuesta única en las evaluaciones va en contra de los Derechos de los Menores y los daña irremediablemente en su desarrollo personal y ciudadano. No podrán nuestros niños y niñas desenvolverse en los contextos sociales a los que se tendrán que enfrentar, una vez que abandonen las aulas.

Por eso los pilares del Proyecto CADE trabajan por el presente educativo y el futuro ciudadano: "En primer lugar el reconocimiento de los niños y niñas como sujetos de derechos de participación y, por tanto, como personas que tienen estatus de ciudadanos. En segundo lugar, la voluntad de poner al servicio de los niños y niñas algunas de las ideas e instrumentos más avanzados en el campo de la ciudadanía, la democracia y el desarrollo humano y social: en particular, las teorías de la democracia deliberativa. Y, en tercer lugar, el convencimiento de que, dado que la tecnología está presente en la forma en la que las democracias se están articulando en la actualidad, no puede haber ejercicio ciudadano sin incorporación de las tecnologías digitales." (Bujanda, M.E. 2005:3) A nosotros además nos sirven para tomar conciencia de que se puede llegar a las soluciones de manera creativa y colectiva. Los docentes pueden reclamar también para sí sus Derechos de Participación ciudadana para hacer efectivos los de sus niños en las aulas y que su ejercicio se pueda extender al resto de sus vidas. Acudimos de nuevo aquí, a las palabras de Felton Earls y 


\section{II: Educación para la diversidad y comunicación}

Maya Carlson "Los niños no pueden prosperar si sus comunidades no son capaces de proporcionar apoyo consistente, sensible y competente, junto con el buen gobierno y servicios humanos. Tampoco las comunidades pueden prosperar si sus miembros más jóvenes no son reconocidos como participantes respetados y activos." (Op.cit. 2011:224)

\section{Nota:}

Quiero expresar aquí mis más efusivas gracias a $\mathrm{M}^{\mathrm{a}}$ Eugenia Bujanda y a Natalia $\mathrm{M}^{\mathrm{a}}$ Rodríguez (Fundación Omar Dengo), y a los doctores Felton Earls y Maya Carlson (Universidad de Harward), sin cuya amable e inestimable ayuda este artículo no hubiera sido posible.

\section{Referencias}

Adell, J. y Castañeda, L. (2012). “Tecnologías emergentes ¿pedagogías emergentes?”, en Hernández Ortega y otros (Coords.). Tendencias emergentes en educación con TIC. Monográfico publicado por Asociación Espiral, Educación y Tecnología, bajo licencia Creative Commons; 13-32.

Alfabetización Mediatica e Informacional. Currículum para profesores. "Alfabetización Mediática e Informacional: curriculum para profesores". Recuperado de http://unesdoc.unesco.org/ images/0021/002160/216099S.pdf

Area Moreira, Manuel (Dir.) (2012). "La visión y opinión del profesorado sobre el Programa Escuela 2.0 en España. Un análisis por comunidades autónomas. Informe final del primer estudio del Proyecto TICSE 2.0 (Tecnologías de la Información y Comunicación en el Sistema Escolar)". Recuperado de http://tece-
du.webs.ull.es/ticse20/media/files/InformeFinal\%20Escuela20-Prof2012.pdf

Bujanda, M.E. (2005). "Ciudadanía y deliberación democrática en la escuela primaria con apoyo de las tecnologías digitales. Una experiencia de investigación y desarrollo curricular". Presentado en la Conferencia "La educación y la formación de una ciudadanía democrática". San José, Costa Rica: 18 y 19 de agosto, 2005. Recuperado de:

https://www.academia.edu/6200459/ Ciudadan\%C3\%ADa_y_deliberaci\%C3\%B3n democr $\%$ C $3 \%$ A 1 tica_en_la_escuela_primaria_con apoyo_de_las_tecnolog\% $3 \%$ ADas_digitales._Una experiencia_de_investigaci\% $\% 3 \% \mathrm{~B} 3 \mathrm{n} \_\mathrm{y}$ _desarrollo curricular

Carlson, M. y Earls, F. (2011). "Adolescents as Deliberative Citizens: Building Health Competence in Local Communities", en The ANNALS of the American Academy of Political and Social Science. vol. 633; 223-242. Recuperado de:

http://ann.sagepub.com/content/633/1/223

Carmona Luque, M. (2011). La Convención sobre Derechos del niño. Instrumento de progresividad en el Derecho Internacional de los Derechos Humanos. Madrid: Dykinson.

Dávila Balsera, P. y Naya Garmendía, L.M. (2006). "La Evolución de los Derechos de la Infancia: Una Visión Internacional", en Encounters on Education Volume 7, Fall; 71-93.

Fonseca, C y Bujanda, M.E. (2011). Promoting children's capacities for active and deliberative citizenship with the help of digital technologies. The experience of CADE Project in Costa Rica (Promocionando las capacidades de los niños para una ciudadanía deliberativa y activa con la ayuda de las 
tecnologías digitales. La experiencia de CADE, Proyecto en Costa Rica). The ANNALS of the American Academy of Political and Social Science. Enero 2011 vol. 633, 1; 243-262.

Fundación Omar Dengo (2005). "Aprender a deliberar para una ciudadanía activa y democrática. Fundamentos teóricos y metodológicos y Guía didáctica para educadores". San José: Fundación Omar Dengo; IDRC. Recuperado de: www.fod.ac.cr/publicaciones Fundación Omar Dengo (2006). "Educación y tecnologías digitales. Cómo valorar su impacto social y sus contribuciones a la equidad". San José: Fundación Omar Dengo.

Fundación Omar Dengo (2009). "Estándares de desempeño de estudiantes en el aprendizaje con tecnologías digitales”. San José: Fundación Omar Dengo. Habermas, J. (1998). Facticidad y validez. Sobre el derecho y el Estado democrático de derecho en término de teoría del discurso. Madrid: Trotta.

Habermas, J. (2010). Teoría de la acción comunicativa, 2 vols. Madrid: Trotta.

Holden, C. y Clough, N. (1998). "The Child Carried on the Back does not Know the Length of the Road. The Teacher's Role in Assinsting Participation". En Holden, C. y Clough, N. (Eds.): Children as Citizens. Education for Participation. Londres. Jessica Kingsley Publishers; 13-28.

Instrumento de Ratificación del Protocolo facultativo de la Convención sobre los Derechos el Niño relativo a un procedimiento de comunicaciones, hecho en Nueva York el 19 de diciembre de 2011. Publicado en: «BOE» núm. 27, de 31 de enero de 2014, páginas 6453 a 6462 (10 págs.) Sección: I. Disposiciones generales Departamento: Jefatura del Estado. Referencia: BOE-A-2014-966. Recuperado de:

https://www.boe.es/diario_boe/txt.php?id=BOEA-2014-966

Instituto Nacional de Tecnologías Educativas y de Formación del Profesorado INTEF, Ministerio de Educación Cultura y Deporte. Gobierno español. Recuperado de:

http://educalab.es/intef/tecnologia

Proyecto Desarrollo de la Capacidad Deliberativa en Escolares (CADE). Fundación Omar Dengo, Costa Rica. Recuperado de:

http://www.fod.ac.cr/index.php?option=com_conten t\&view $=$ article $\&$ id=81\&Itemid=160\&area $=9 \&$ proye cto $=30$

https://www.fod.ac.cr/cade/

Santos Guerra, M. Á. (2014). "Las mil caras de la violencia contra el niño y la niña”. En Jiménez Hernández, A. S. y otros (Coords.) Infancia en Contextos de Riesgo. XXV Años de la Convención sobre los Derechos del Niño. Granada: GEU; 211-223.

Sen, A. (1999). Development as Freedom. New York: Alfred A. Knopf.

UNICEF (2009). The State of the World's Children. Special Edition. Celebrating 20 Years of the Convention on the Rights of the Child. New York: UNICEF. Recuperado de: http://www.unicef.org/publications/ 